\title{
Protein-mediated lipid transfer
}

\author{
The effects of lipid-phase transition and of charged lipids
}

\begin{abstract}
You-Han XÜ,* Klaus GIETZEN, $†$ Hans-Joachim GALLA* $\ddagger$ and Erich SACKMANN§ *Department of Biophysics and $\dagger$ Department of Pharmacology and Toxicology, University of Ulm, Oberer Eselsberg, D-7900 Ulm (Donau), Federal Republic of Germany, and §Department of Physics E22, Technical University of Munich, D-8046 Garching, Federal Republic of Germany
\end{abstract}

(Received 11 November 1982/Accepted 7 February 1983)

\begin{abstract}
The protein-mediated phospholipid exchange between small unilamellar vesicles was investigated by fluorescence polarization measurements with diphenylhexatriene as optical probe. Thermotropic phase-transition measurements were taken after mixing two vesicle preparations of distinct and different phase-transition temperatures or having different states of charge. From the heights of each phase-transition step, we were able to follow the lipid-exchange process in the presence, as well as in the absence (natural exchange), of so-called transfer protein isolated from beef liver. A strong enhancement of the lipid transfer was observed at the corresponding lipid-phase-transition temperature, which is explained by the presence of fluctuating fluid and ordered domains co-existing at the lipid-phase-transition temperature. A unidirectional lipid transfer of the neutral component was observed between negatively charged phosphatidic acid and neutral phosphatidylcholine vesicles. Fluorescence polarization measurements showed the disappearance of the phosphatidylcholine phase transition, whereas the phosphatidic acid phase transition broadened and its phase transition temperature became lower.
\end{abstract}

It has been known for several years that the exchange of phospholipids between natural membranes occurs via cytosolic proteins (Wirtz \& Zilversmith, 1969). These so-called phospholipidtransfer proteins isolated from liver and other tissues (Wirtz, 1974; Kamp \& Wirtz, 1974; Zilversmit \& Johnson, 1975) bind specific classes of phospholipids and catalyse their transfer between membrane interfaces (Zilversmit \& Hughes, 1976, 1977). Non-specific exchange proteins from rat or beef liver have been found to catalyse the transfer of phosphatidylcholines, phosphatidylethanolamines, phosphatidylserines or phosphatidylinositols between rat liver microsomal membranes (Crain \&

Abbreviations used: DMPC (dimyristoyl)phosphatidylcholine (dimyristoylglycerophosphocholine); DPPC, (dipalmitoyl)phosphatidylcholine (dipalmitoylglycerophosphocholine); DSPC (distearoyl)phosphatidylcholine (distearoylglycerophosphocholine); DMPA, (dimyristoyl)phosphatidic acid (dimyristoylglycerophosphate).

$\ddagger$ To whom correspondence and reprint requests should be sent at: Department of Biochemistry, Technische Hochschule, D-6100 Darmstadt, Federal Republic of Germany.
Zilversmit, 1980) and between erythrocytes and unilamellar vesicles. Moreover specific proteins, for example a phosphatidylcholine exchange protein has been isolated from beef liver and was used to investigate the protein-mediated transfer of phosphatidylcholines in natural membranes and artificial phospholipid vesicles (Wirtz et al., 1976; Kasper \& Helmkamp, 1981). A kinetic analysis of the interaction between phosphatidylcholine-exchange protein and phosphatidylcholine vesicles carried out by Van den Besselar et al. (1975) demonstrated the formation of a strong protein-membrane complex. This complex was even stronger if the vesicles contained negatively charged phosphatidic acid (Wirtz et al., 1979).

In the present study we have investigated the lipid-transfer process in phospholipid bilayer vesicles by the use of fluorescence polarization measurements. It was shown recently (Xü et al., 1983) that it is possible to quantify the amount of lipid in a given state from the thermotropic phase-transition curve. We can now follow directly the transfer process between phospholipid vesicles that exhibit different phase transitions. This method has been applied to

Vol. 213 
investigate the temperature dependence of the lipid-transfer process in the phase transition region of the corresponding lipids. A unidirectional transfer could be shown between phosphatidylcholine and phosphatidic acid vesicles.

\section{Materials and methods}

Phospholipid-transfer protein was isolated from fresh beef liver by a slight modification (Xü et al., 1983) of the standard purification method (Kamp \& Wirtz, 1974). Protein concentration was determined by the method of Lowry et al. (1951).

DMPC, DPPC and DSPC, as well as DMPA, were obtained from Fluka (Neu-Ulm, Germany) and used without further purification.

Lipids and $1 \%$ diphenylhexatriene (Fluka) were dissolved in chloroform and evaporated under $\mathrm{N}_{2}$ in a flask to yield a film on the flask wall. Buffer $(0.1 \mathrm{M}$-sodium phosphate, $\mathrm{pH} 7)$ was added to the dried film to make up a lipid concentration of $0.5 \mathrm{mg} / \mathrm{ml}$. Sonification was performed with a Branson Ultrasonifier $30 \mathrm{~W}$ at a temperature $10^{\circ} \mathrm{C}$ above the corresponding lipid-phase-transition temperature until clear dispersions of small unilamellar vesicles were obtained. In a previous study (Xü $e t$ al., 1983) describing these techniques we did not use strictly unilamellar vesicles, leading to a lower relative amount of transferred lipid.

Fluorescence polarization measurements to obtain the phase-transition curves were performed automatically with increasing temperature as described previously (Hartmann et al., 1978; Xü et al., 1983). The heating rate was $3^{\circ} \mathrm{C} / \mathrm{min}$. The vesicles were mixed and incubated for a given time in the presence of transfer protein at a concentration of $20 \mu \mathrm{g} / \mathrm{ml}$ of lipid dispersion. The incubation temperature was varied. Samples were measured immediately and inhibition of the protein activity after the incubation time was not necessary. Only negligible changes occurred during the measurement of the phasetransition curves.

\section{Results}

\section{Temperature-dependence of the transfer process}

Thermotropic phase transition curves of vesicle mixtures containing equal amounts of DPPC and DSPC vesicles and $20 \mu \mathrm{g}$ of transfer protein per $\mathrm{ml}$ of lipid dispersion are shown in Fig. 1 for different incubation temperatures, together with a control measurement. The control curve is measured immediately after mixing of the two vesicle preparations in the presence of exchange protein but without incubation. The almost horizontal course of the change in fluorescence polarization between 42 and $50^{\circ} \mathrm{C}$ signifies that only minor lipid transfer occurred during the measurement of the phase-

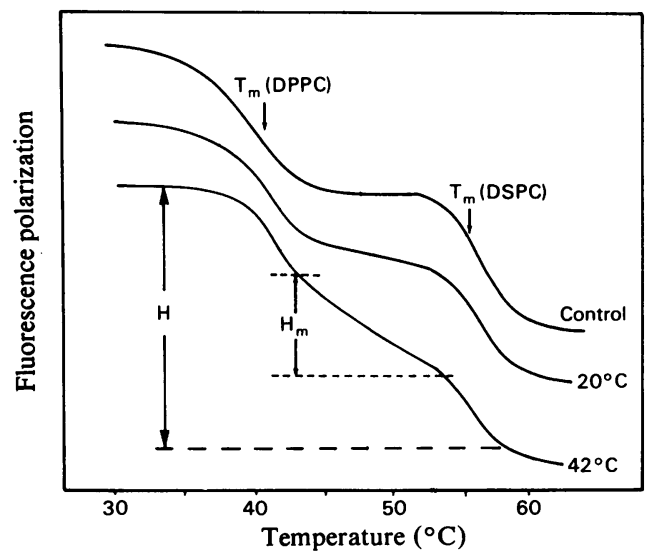

Fig. 1. Phase-transition curves of mixtures of DPPC and $D S P C$ vesicles after addition of transfer protein and immediately measured (control) and after incubation for $30 \mathrm{~min}$ at 20 or $42^{\circ} \mathrm{C}$ in the presence of $20 \mu \mathrm{g} / \mathrm{ml}$ of phosphatidylcholine-transfer protein

Diphenylhexatriene was used as optical probe to measure the fluorescence polarization with increasing temperature. The phase transition temperatures of either lipid are marked by $T_{m}$. The step height ratio $\mathrm{H}_{\mathrm{m}} / \mathrm{H}$ is taken as a measure of the amount of transferred lipid.

transition curve. Phase-transition curves taken after incubation for $30 \mathrm{~min}$ at 20 or $42^{\circ} \mathrm{C}$ respectively, are typical examples for different transfer activities. Beside the phase transitions of remaining pure DPPC or DSPC lipid phase, one clearly observes melting of a mixed lipid phase between 42 and $50^{\circ} \mathrm{C}$. The step height ratio, $\mathrm{H}_{\mathrm{m}} / \mathrm{H}$, of the change in fluorescence polarization of this intermediate range with respect to the total height of the phase transition was used to quantify the relative amount of transferred lipid. Incubation at $42^{\circ} \mathrm{C}$ is accompanied by a considerably higher transfer compared with that at $20^{\circ} \mathrm{C}$.

The transfer activity evaluated from the phasetransition curves obtained by fluorescence polarization is given in Fig. 2 as a function of the incubation temperature. The incubation time was $30 \mathrm{~min}$ and the transfer protein concentration was $20 \mu \mathrm{g} / \mathrm{ml}$. The temperature range covers the corresponding lipid-phase-transition temperatures, which are $40^{\circ} \mathrm{C}$ for DPPC and $55^{\circ} \mathrm{C}$ for DSPC vesicles. The low activity at low temperatures increases to a first maximum of the transfer activity at $42^{\circ} \mathrm{C}$. The decrease in the amount of lipid transfer with increasing temperature up to $50^{\circ} \mathrm{C}$ is followed by a second maximum at $52^{\circ} \mathrm{C}$. Evidence is given that the protein-mediated lipid transfer is controlled by the physical state of the phospholipid vesicles. The transfer activity is highest at the phase transition temperature of either lipid. 


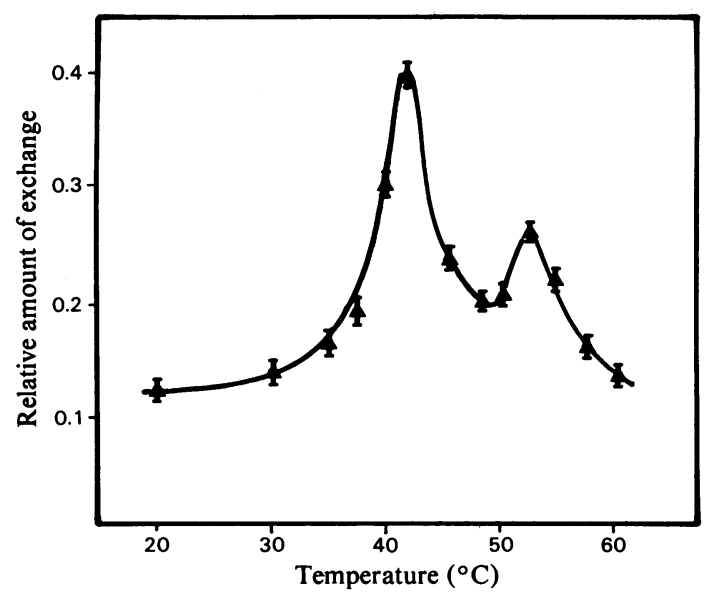

Fig. 2. Temperature-dependence of the exchange activity of phosphatidylcholine-exchange protein in a mixture of DPPC and DSPC vesicles

The maximum at $42^{\circ} \mathrm{C}$ agrees well with the phase-transition temperature of DPPC. A second maximum at $52^{\circ} \mathrm{C}$ is observed at the phase transition of DSPC vesicles. The error bars are given in the Figure $(n=4)$.

To exclude temperature-dependent denaturation of the transfer protein itself we pretreated the protein for $30 \mathrm{~min}$ at temperatures up to $70^{\circ} \mathrm{C}$. The pretreated protein was added to mixtures of DPPC and DSPC vesicles and the transfer process was determined after $30 \mathrm{~min}$ of incubation at $42^{\circ} \mathrm{C}$. Fig. 3 shows that pretreatment up to $55^{\circ} \mathrm{C}$ does not change the transfer potency of the protein. Application of higher temperatures leads to a continuous decrease in transfer potency of the pretreated protein.

Protein-mediated lipid transfer is expected to occur much faster than natural lipid transfer. To exclude a concomitant transfer in the absence of protein we determined also the natural transfer by recording the transition curves of mixtures of DPPC and DSPC vesicles immediately after mixing and after 3 or $7 \mathrm{~h}$ of incubation at $60^{\circ} \mathrm{C}$ (results not shown). Note that the incubation temperature of $60^{\circ} \mathrm{C}$ is well above the lipid-phase-transition temperatures of the phosphatidylcholines. A small exchange of lipids was observed within $7 \mathrm{~h}$. However, within $3 \mathrm{~h}$ the lipid exchange in the absence of protein is negligible.

\section{The effect of negatively charged lipids}

Phase-transition curves of vesicle mixtures of DMPC and DMPA in phosphate buffer, $\mathrm{pH} 9.0$, were taken at different incubation times in the presence of exchange protein (Fig. 4). The pH-value of the solution was well above the $\mathrm{p} K$ value of the protein ( $\mathrm{p} K$ approx. 6), which means that the protein, as well as the phosphatidic acid vesicles, is

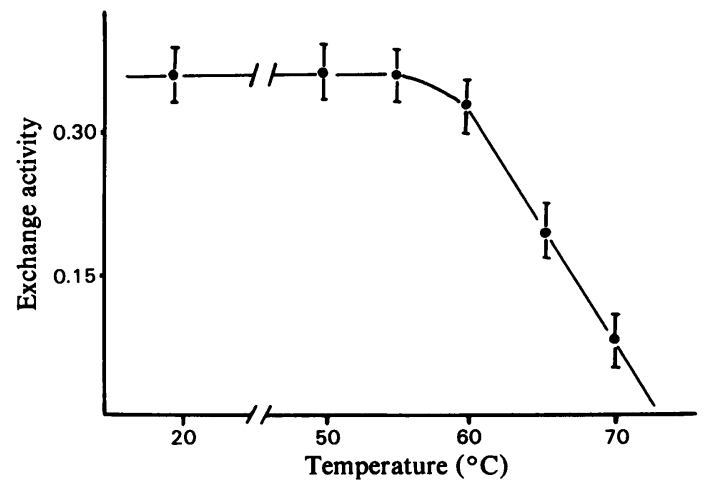

Fig. 3. Heat effect on the protein activity

The protein solution was preheated for $30 \mathrm{~min}$ at the temperature given on the abscissa of the graph. The exchange activity was determined from phase-transition curves after incubation for $30 \mathrm{~min}$ at $42^{\circ} \mathrm{C}$ in the presence of the preheated protein. No change in protein activity was observed up to $55^{\circ} \mathrm{C}$. Higher temperatures lead to a loss in activity. Again DPPC and DSPC vesicles were used.

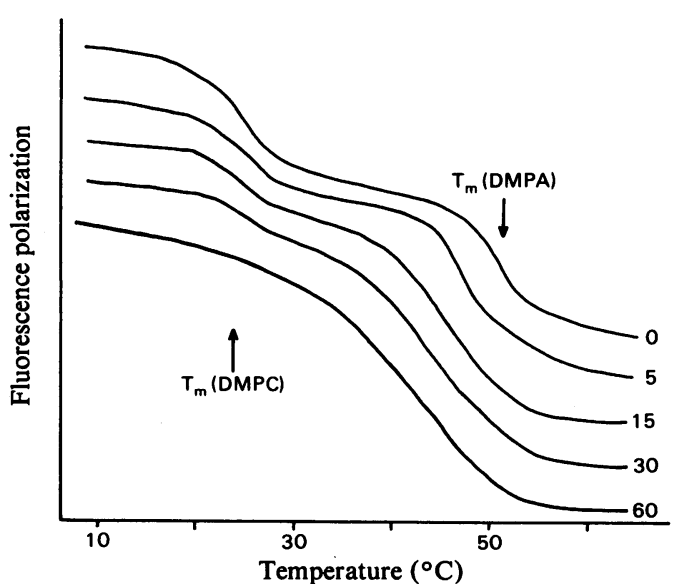

Fig. 4. Phase-transition curves of a mixture of DMPC and DMPA in the presence of exchange protein taken at pH 9

The protein concentration was $15 \mu \mathrm{g} / \mathrm{ml}$; the incubation temperature was $45^{\circ} \mathrm{C}$. The number by each curve indicates the incubation time (min).

negatively charged. At time zero the phase-transition steps of DMPC and DMPA are very well separated. The intermediate temperature range between 30 and $45^{\circ} \mathrm{C}$ has a small slope that is also present in the absence of exchange protein if DMPC vesicles are used. With increasing incubation time we observed a relative increase in the phase-transition step of the phosphatidic acid vesicles, whereas the phasetransition step of DMPC vesicles diminishes. Moreover the phase-transition temperature of DMPA vesicles is lowered and broadened, showing the incorporation of low-melting DMPC into DMPA 
vesicles. The disappearance of the DMPC phase transition clearly demonstrates that phosphatidic acid molecules are not transferred into phosphatidylcholine vesicles, whereas phosphatidylcholine is transferred into phosphatidic acid vesicles at pH 9.0.

\section{Discussion}

An interesting new observation in the present work is the temperature-dependence of the exchange process between DPPC and DSPC vesicles. The exchange activity is most pronounced at the phasetransition temperature of either lipid, even if the second lipid is in the quasicrystalline state. Below and above the lipid-phase transition, we observed a lowered exchange activity, which is increased again at the phase transition of the second type of lipid vesicle. Obviously the properties of the membrane lipid that is undergoing the phase transition are of importance for the transfer process.

The possible physiological significance of lipidphase transitions in triggering membrane functions has been emphasized in the last decade (Träuble, 1972). The lipid-phase transition is considered to have a continuous nature (Lee, 1975) caused by defect structures in the gel-phase. The defects may be grain boundaries that separate two crystals or grains that differ in orientation (Lee, 1977). Highangle grain boundaries may form vacancies and thus create volumes of disorder in an otherwise crystalline phase. More generally the interfacial lipid in the boundary regions between fluid and ordered phases that co-exist at the phase-transition temperature could have important effects on membrane function owing to the mismatch in molecular packing (Marsh et al., 1976). Several authors (Papahadjopoulos et al., 1973; Wu \& McConnell, 1973) have reported sharp peaks in the ion permeability at the phase transition, with lower permeabilities below and above the lipid-phase transition. Marsh et al. (1976) measured a maximum in the permeability of a cation spin-label at the lipid-phase transition, which could be quantitatively calculated from the fraction of interfacial boundary lipid at various stages throughout the phase transition. The fraction of interfacial molecules is maximal at the phase transition and smaller in the ordered and in the fluid state.

Our results provide evidence that interfacial lipids at the boundaries of regions of fluid and ordered phase may be especially susceptible to lipid transfer. Recently we observed in an electron-microscopy study the preferential transfer of lipids from a vesicle in the fluid state into defect structures of a vesicle in the $\mathrm{P}_{\beta}$,-phase (Xü et al., 1983). It is noteworthy that defects are stabilized and broadened in the presence of small amounts of impurities (Sackman et al., 1981).

In this sense incorporation of a different lipid molecule can be considered as an impurity. The transfer process may start at grain boundaries but is expected to cover the whole vesicle, as was observed by electron microscopy (Xü et al., 1983).

The second noteworthy observation of the present paper is the transfer of charged lipids. Here we used phosphatidic acid as an example. The phasetransition curves shown in Fig. 4 do not allow quantification of the amount of transferred lipid. However, qualitatively we find a unidirectional transfer of the uncharged component into the charged vesicles. This conclusion was drawn from the disappearance of the DMPC phase transition and the lowered and broadened DMPA phase transition at $\mathrm{pH}$ 9.0.

This work was supported by Deutsche Forschungsgemeinschaft (contracts Ga 233 and Sa 246). Y.-H.X. was supported by a grant from the Government of the People's Republic of China.

\section{References}

Crain, W. C. \& Zilversmit, D. B. (1980) Biochemistry 19, 1440-1447

Hartmann, W., Galla, H.-J. \& Sackmann, E. (1978) Biochim. Biophys. Acta 510, 124-139

Kamp, H. H. \& Wirtz, K. W. A. (1974) Methods Enzymol. 32, 140-146.

Kasper, A. M. \& Helmkamp, G. M. (1981) Biochemistry 20, 146-151

Lee, A. G. (1975) Prog. Biophys. Mol. Biol. 29, 3-9

Lee, A. G. (1977) Biochemistry 16, 835-841

Lowry, O. H., Rosebrough, N. J., Farr, A. L. \& Randall, R. J. (1951) J. Biol. Chem. 193, 265-275

Marsh, D., Watts, A. \& Knowles, P. (1976) Biochemistry $15,3570-3578$

Papahadjopoulos, D., Jacobson, K., Nir, S. \& Isac, T. (1973) Biochim. Biophys. Acta 311, 330-348

Sackmann, E., Gebhardt, E. \& Rüppel, D. (1981) in Springer Series in Chemical Physics (Helfrich, W. \& Heppke, G., eds.), vol. 11, pp. 309-326, Springer, Berlin, Heidelberg and New York

Träuble, H. (1972) Biomembranes 3, 197-206

Van den Besselar, A. M. H. P., Helmkamp, G. M. \& Wirth, K. W. A. (1975) Biochemistry 14, 1952-1959

Wirtz, K. W. A. (1974) Biochim. Biophys. Acta 344, 95-117

Wirtz, K. W. A. \& Zilversmit, D. B. (1969) Biochim. Biophys. Acta 193, 105-116

Wirtz, K. W. A., Geurts van Kessel, W. S. M., Kamp, H. H. \& Demel, R. A. (1976) Eur. J. Biochem. 61, 515-523

Wirtz, K. W. A., Vriend, G. \& Westermann, J. (1979) Eur. J. Biochem. 94, 215-221

Wu, S. H. \& McConnell, H. M. (1973) Biochem. Biophys. Res. Commun. 55, 484-491

Xü, Y. H., Rüppel, D., Ziegler, H., Hartmann, W. \& Galla, H.-J. (1982) Biochim. Biophys. Acta 689, 437-443

Xü, Y. H., Gietzen, K., Galla, H.-J. \& Sackmann, E. (1983) Biochem. J. 209, 257-260

Zilversmit, D. B. \& Hughes, M. E. (1976) Methods Membr. Biol. 7, 211-259

Zilversmit, D. B. \& Hughes, M. E. (1977) Biochim. Biophys. Acta 469, 99-110

Zilversmit, D. B. \& Johnson, L. W. (1975) Methods Enzymol. 35, 262-269 